\title{
Traumatismo cranioencefálico e sua associação com uso de canabinoides e cocaína: experiência de hospital universitário brasileiro
}

\author{
José Weber Vieira de Faria", Cláudio Márcio Silva de Souza², Sérgio de Andrade \\ Nishioka ${ }^{3}$, Guilherme Leonel Arbex 4 , Gustavo Gil Alarcão ${ }^{4}$, Wender Barbosa de Freitas ${ }^{4}$
}

Serviço de Neurocirurgia do Hospital de Clínicas da Faculdade de Medicina da Universidade Federal de Uberlândia, MG.

\section{RESUMO}

Objetivo: Este estudo objetivou identificar aspectos da epidemiologia dos traumatismos cranioencefálicos (TCEs) moderados e graves dos pacientes admitidos no Hospital de Clínicas da Universidade Federal de Uberlândia, bem como sua associação com consumo de cocaína e canabinoides. Métodos: Para isso, foram coletados dados prospectivos sobre 139 vítimas assistidas nesse hospital, todas com idade igual ou superior a 18 anos. Os acidentes de transporte responderam pelo maior número de vítimas, seguidos daquelas pessoas que sofreram quedas acidentais. Resultados: Do total de pacientes avaliados, 84 (com casos de TCE moderado, grave e mesmo leve) tiveram amostra de urina coletada para detecção de maconha e cocaína. Os exames foram positivos para maconha em $8,2 \%$ dos pacientes e em 13,9\% para cocaína. Conclusão: Não foram vistas diferenças significativas no tocante ao sexo e à idade, bem como à atividade realizada no momento do trauma. A maioria dos atendimentos desses pacientes, no entanto, se deu no período noturno. A alta frequência de consumo de substâncias ilícitas associada aos TCEs evidencia a importância de se incluírem esses dados quando da realização de estudos acerca desse tipo de traumatismo, bem como aponta para um problema de saúde pública.

\section{PALAVRAS-CHAVE}

Traumatismos encefálicos, cocaína, canabinoides, transtornos relacionados ao uso de substâncias.

\section{ABSTRACT}

Traumatic brain injury and its association to marijuana and cocaine use: a Brazilian university hospital experience

Objective: This study aimed at identifying epidemiological aspects of moderate and severe traumatic brain injury (TBI) of patients who were admitted at the Clinics Hospital of the Federal University of Uberlândia, as well as its association with marijuana and cocaine intake. Methods: Prospective data were collected from 139 assisted patients, all of them aged 18 or more years. Traffic accidents were responsible for the greatest number of victims, followed by accidental drops. Results: Eighty-four patients (including moderate, severe and even light TBI) had a urine sample evaluated for the presence of marijuana and cocaine. Marijuana positive tests were found in $8.2 \%$ of patients, and there were 13.9\% for cocaine. Conclusion: No significant differences were seen concerning age and sex, neither for the activity performed at the trauma time. However more patients were admitted at the hospital at night time. The high frequency of consumption of these illegal stuff associated with TBI shows the importance of having this information while performing trials about this kind of trauma. It also indicates this is public health problem.

\section{KEY-WORDS}

Brain injuries, cocaine, cannabinoids, substance-related disorders.

1. Chefe do Serviço de Neurocirurgia da Faculdade de Medicina da Universidade Federal de Uberlândia (UFU), Uberlândia, MG, médico pesquisador e pós-graduando da Universidade de São Paulo (USP), SP, Brasil.

2. Médico-residente do Serviço de Neurocirurgia da Faculdade de Medicina da UFU, MG, Brasil.

3. Professor-assistente do Departamento de Clínica Médica da Faculdade de Medicina da UFU, MG, Brasil.

4. Médico-assistente do Hospital de Clínicas da Faculdade de Medicina da UFU, MG, Brasil. 


\section{Introdução}

Entre as mais temidas de todas as injúrias agudas, figura com grande importância a injúria cerebral. Ela responde não somente como causa de morte, mas também como causa de sequelas funcionais, psicológicas e fisiológicas permanentes. ${ }^{15} \mathrm{O}$ traumatismo cranioencefálico (TCE) é uma das principais causas de injúria cerebral aguda. É também um problema de saúde pública ${ }^{7,6,10,20,24,28}$ que atinge tanto os países desenvolvidos como aqueles em desenvolvimento. Assim sendo, entender a epidemiologia dos TCEs passa a configurar como um importante instrumento para implementação de ações que visem a reduzir a incidência desses traumatismos. E nesse contexto enquadra-se o conhecimento regionalizado da epidemiologia.

Quando se trata de TCE, esse pode ser classificado segundo algumas variáveis, quais sejam: intenção do trauma, ${ }^{3}$ em intencional, não intencional ou intenção indeterminada; causa da injúria, situação em que se deve destacar a causa externa e desmembrá-la em acidentes de transportes, agressões físicas, quedas, atividades esportivas, entre outros; e também a severidade do trauma, que em países desenvolvidos tem sido avaliada por meio de escalas como a Escala de Injúria Abreviada (Abbreviated Injury Scale - AIS) e o Escore de Severidade da Injúria (Injury Severity Score - ISS) ${ }^{3}$ e, mais recentemente, o Novo Escore de Severidade da Injúria (New Injury Severity Score - NISS). Essas escalas têm importância no paciente com múltiplos traumas, em virtude de facilitar comparações. ${ }^{2}$ É ainda de grande valia para avaliação da severidade do trauma o nível de consciência do paciente, sendo este avaliado por meio da Escala de Coma de Glasgow (ECG), ${ }^{14}$ que, embora possa apresentar algumas dificuldades e até limitações em sua utilização, ${ }^{8,12,30}$ é normalizada pela Sociedade Brasileira de Neurocirurgia em sua publicação de normas e diretrizes para o TCE. ${ }^{1}$

A análise de determinantes específicos para diferentes tipos de injúria caracteriza-se por ser um elemento essencial para o controle dessa injúria. Tipos específicos de injúria tendem a ocorrer mais frequentemente em certos subgrupos de uma população em virtude de suas características pessoais ou por sua maior exposição a fatores de risco ambientais, de equipamento ou das atividades que exercem. ${ }^{3}$ No que diz respeito à idade, a maioria dos casos de TCE, independentemente de sua gravidade, ocorre em adolescentes e adultos jovens. ${ }^{15}$ Há um predomínio do TCE no sexo masculino, ${ }^{4,6,8,11,19,20,22,23}$ e também há relatos na literatura de que esse tipo de trauma ocorra com maior frequência em pessoas de mais baixa condição social. ${ }^{17,25}$

A associação entre TCE e o consumo de drogas ilícitas é ainda um campo a ser mais bem explorado.
O primeiro passo na determinação da importância da intoxicação por drogas na etiologia e evolução do trauma é estabelecer a presença dessas drogas em pacientes com vários tipos de trauma, fatais e não fatais. Pouco se sabe sobre o uso de drogas em países em desenvolvimento. A correlação do uso da droga com a concentração nos fluidos corporais necessita de melhores estudos e acredita-se que a sua presença, independentemente da concentração, impede a função psicomotora. ${ }^{9} \mathrm{O}$ uso agudo pode também gerar efeitos diferentes em relação ao uso continuado das drogas. Os exames atuais são semiquantitativos, indicando apenas a exposição prévia, não se conhecendo os níveis de intoxicação.

O nível da droga na urina não se correlaciona com o grau de impedimento, e os sintomas são frequentemente mascarados pelo uso simultâneo do álcool. Além do mais, a presença de metabólitos tanto de maconha como de cocaína na urina não é indicativo de consumo recente da substância. ${ }^{29}$

Buscou-se neste estudo conhecer o perfil epidemiológico do traumatismo cranioencefálico moderado e grave atendido no HC da UFU e sua associação com o uso de cocaína e canabinoides, bem como os determinantes específicos para a injúria cerebral. Dessa forma, tentou-se obter dados ou elementos essenciais para um possível futuro controle dessa injúria, pois, ao se identificarem determinantes suscetíveis à modificação, poder-se-á desenvolver intervenções apropriadas, que contribuam para o controle desse problema.

\section{Casuística e métodos}

Foi realizado um estudo prospectivo em 139 pacientes com diagnóstico de TCE grave e moderado e com idade igual ou superior a 18 anos, atendidos na Unidade de Emergência (UE) do Hospital de Clínicas (HC) da Universidade Federal de Uberlândia (UFU), localizada na cidade de Uberlândia (500.488 hab), estado de Minas Gerais, durante o período de janeiro a dezembro de 2002.

Conceitualmente, foram considerados portadores de TCE aqueles traumatizados que se enquadraram na definição proposta por Jennett $e$ MacMillan ${ }^{13}$, qual seja, pacientes que apresentavam uma ou mais das seguintes condições: 1) história definida de golpe na cabeça; 2) laceração de couro cabeludo ou na região frontal; 3) alteração da consciência, independentemente de sua duração.

Quanto à severidade do trauma, foram considerados como TCE grave e moderado aqueles pacientes com nível de consciência de 3 a 8 pontos e de 9 a 12 pontos na ECG, respectivamente. A referida escala foi aplicada logo após o atendimento inicial ao paciente politrau- 
matizado na UE. Foram também incluídos aqueles pacientes que nas primeiras 24 horas evoluíram com rebaixamento no nível de consciência para os valores entre 3 e 12 pontos na ECG.

Os dados foram coletados por meio de entrevista com familiares, amigos, socorristas, acompanhantes e com os profissionais da saúde que prestaram atendimento ao paciente no hospital, uma vez que os pacientes não tinham condições para fornecê-los. Foi utilizado um protocolo previamente elaborado, contendo informações relativas à identificação do paciente, características do trauma, natureza e causa da injúria e fatores relacionados. Os dados foram armazenados em um banco de dados Microsoft Access ${ }^{\circledR}$ com interface desenvolvida em Delphi 6.0, elaborado, especificamente, para o estudo a partir do protocolo.

Para avaliação da presença de metabólitos da cocaína e canabinoides na urina, formaram-se dois grupos de pacientes: o primeiro grupo constou de uma amostra de 56 pacientes, atendidos de forma sequencial com ECG de 3 a 12 pontos (TCE grave e moderado), em um período de quatro meses consecutivos, escolhidos por conveniência, no período de agosto a novembro de 2002; o segundo, de 29 pacientes com ECG de 13 a 15 (TCE leve), cujas amostras foram colhidas em duas semanas não consecutivas, escolhidas de forma aleatória (por sorteio simples) dentro do período da coleta das amostras para os pacientes com TCE grave e moderado, sendo analisadas as amostras de um a cada dois pacientes, de forma alternada, de acordo com a chegada a UE.

O termo de consentimento esclarecido foi aplicado a todos os pacientes conscientes (traumas leves) e aos responsáveis pelos pacientes com traumas graves e moderados. O protocolo para este estudo foi aprovado pelo Comitê de Ética em Pesquisa da UFU.

Teste laboratorial: Usando o Axsym ${ }^{\oplus}$ System do laboratório Abbott e o ensaio $\mathrm{AxSYM}^{\circledR}$ canabinoides e o ensaio $\mathrm{AxSYM}^{\circledast}$ metabólitos da cocaína, realizou-se a detecção preliminar e semiquantitativa de canabinoides e metabólitos da cocaína na urina humana. Foi utilizado um cutoff de 50,00 ng/ml para os ensaios de canabinoides e um cutoff de $300,00 \mathrm{ng} / \mathrm{ml}$ para ensaio de metabólitos da cocaína. As informações foram utilizadas de forma a não se identificar o paciente, sendo armazenadas diretamente no banco de dados elaborado para o trabalho.

Métodos estatísticos: Comparações entre variáveis numéricas e contínuas realizadas pelos teste $t$ ou, quando apropriado, pelo teste de Mann-Whitney. Também foram calculadas razões de risco, com intervalos de confiança de $95 \%$. Para os cálculos estatísticos, utilizou-se o software Epi-Info 6.04d (Centers for Disease Control and Prevention, Atlanta, CA, EUA).

\section{Resultados}

A análise epidemiológica dos pacientes vítimas de TCE mostrou que o número de atendimentos diários aumentou progressivamente no final de semana, atingindo um pico ao sábado. Evidenciou-se que aproximadamente $60 \%$ dos pacientes com TCE grave e moderado chegaram na sexta-feira, sábado e domingo (42,9\% dos dias da semana). Observou-se aos sábados uma maior prevalência, sendo admitidos $24,5 \%$ dos pacientes. Quanto ao horário de entrada no serviço, a maior frequência de chegada foi no período das 16 às 23 horas $(52,5 \%)$.

Do total de casos com TCE grave e moderado, 119 $(85,6 \%)$ foram do sexo masculino e $20(14,4 \%)$ do sexo feminino, havendo um predomínio do sexo masculino em todas as faixas etárias em uma relação homens/ mulheres de 5,95:1,00. A idade dos pacientes variou de 18 a 89 anos, com uma média de 40,73 anos (Figura 1).

A maior frequência de TCE grave e moderado ocorreu com vítimas de acidentes de transporte, principalmente na faixa etária de 30 a 39 anos. Para agressões, observou-se que a faixa etária de maior frequência foi dos 20 aos 29 anos, com $47,8 \%$ das ocorrências, e todas no sexo masculino. A maior frequência de quedas ocorreu na faixa etária acima dos 60 anos (33,3\%), sendo que a razão homens/mulheres foi de 3:1. A maior frequência de agressões foi observada na faixa de 20 a 29 anos $(47,8 \%)$, sendo $100 \%$ do sexo masculino.

Oitenta e cinco pacientes com diagnóstico de TCE, sendo 56 graves e moderados e 29 leves, submeteram-se à análise laboratorial para detecção do uso de derivados canabinoides e metabólitos da cocaína na urina. A idade desses pacientes variou de 18 a 85 anos (mediana de 37 anos para TCE grave e moderado e 33,5 anos para TCE leve, com média de 40,6 anos e 34,8 anos, respectivamente); $89,2 \%$ dos TCEs leves e $76,8 \%$ dos TCEs graves e moderados foram pertencentes à faixa etária compreendida entre 18 e 49 anos (Figura 2). Não houve diferença significativa na comparação entre sexo e média de idade, em relação à severidade do trauma, sendo a média de idade entre os TCEs graves e moderados de 40,6 anos e entre os TCEs leves de 34,8 anos.

Observou-se nos pacientes avaliados quanto ao uso de drogas uma prevalência de sete casos $(8,3 \%)$ com exame positivo para maconha e $11(13,9 \%)$ para cocaína. A maior prevalência de cocaína foi dos 20 aos 29 anos e de maconha dos 50 aos 59 anos, não sendo observado qualquer exame positivo em pacientes com mais de 60 anos. Foi também observada maior prevalência de exames positivos para cocaína e maconha em indivíduos do sexo masculino e entre aqueles que se encontravam empregados. 


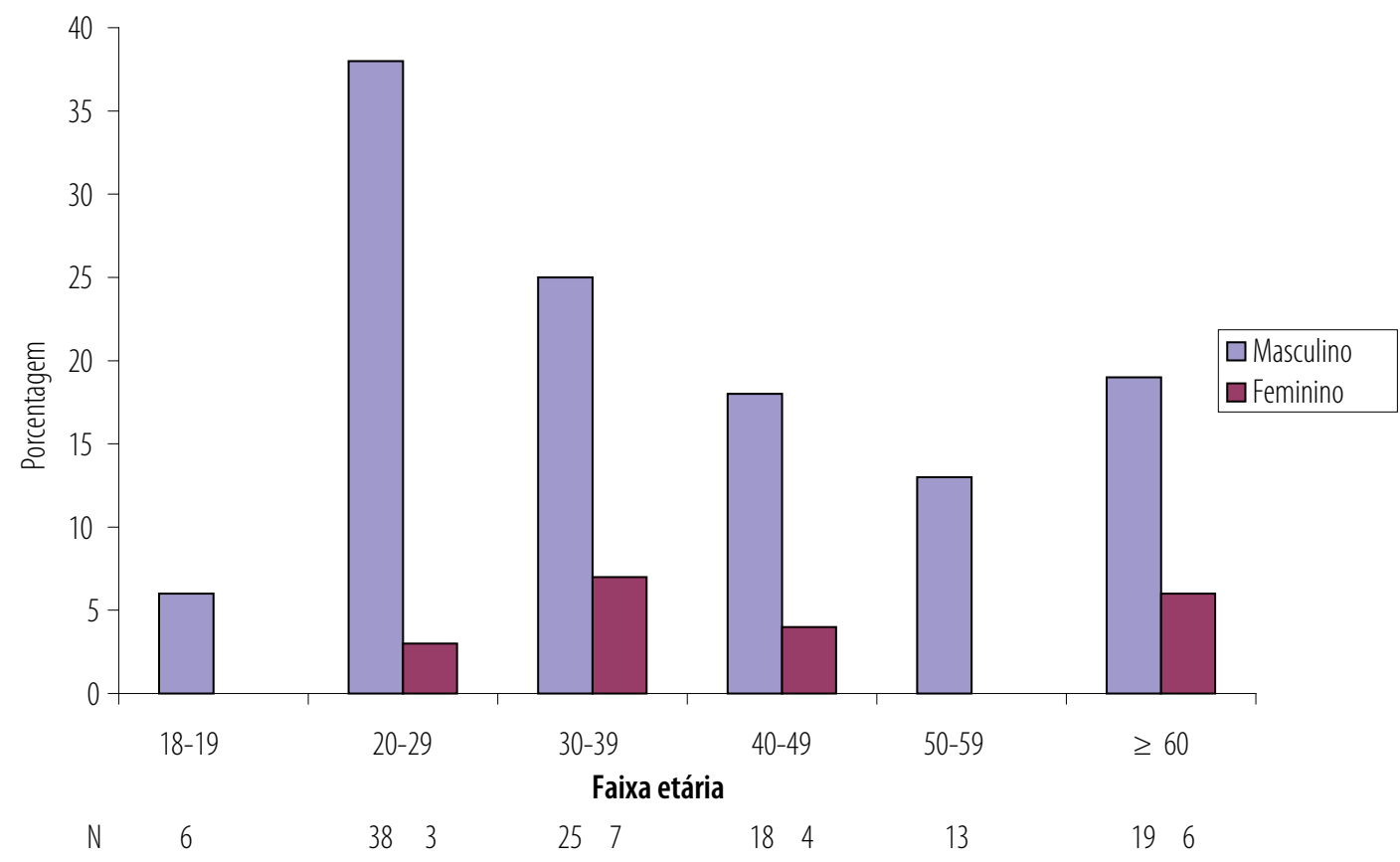

Figura 1 - Distribuição etária e por sexo dos pacientes com TCE grave e moderado $(n=139)$ com idade igual ou superior a 18 anos atendidos na UE do HC da UFU no ano de 2002.

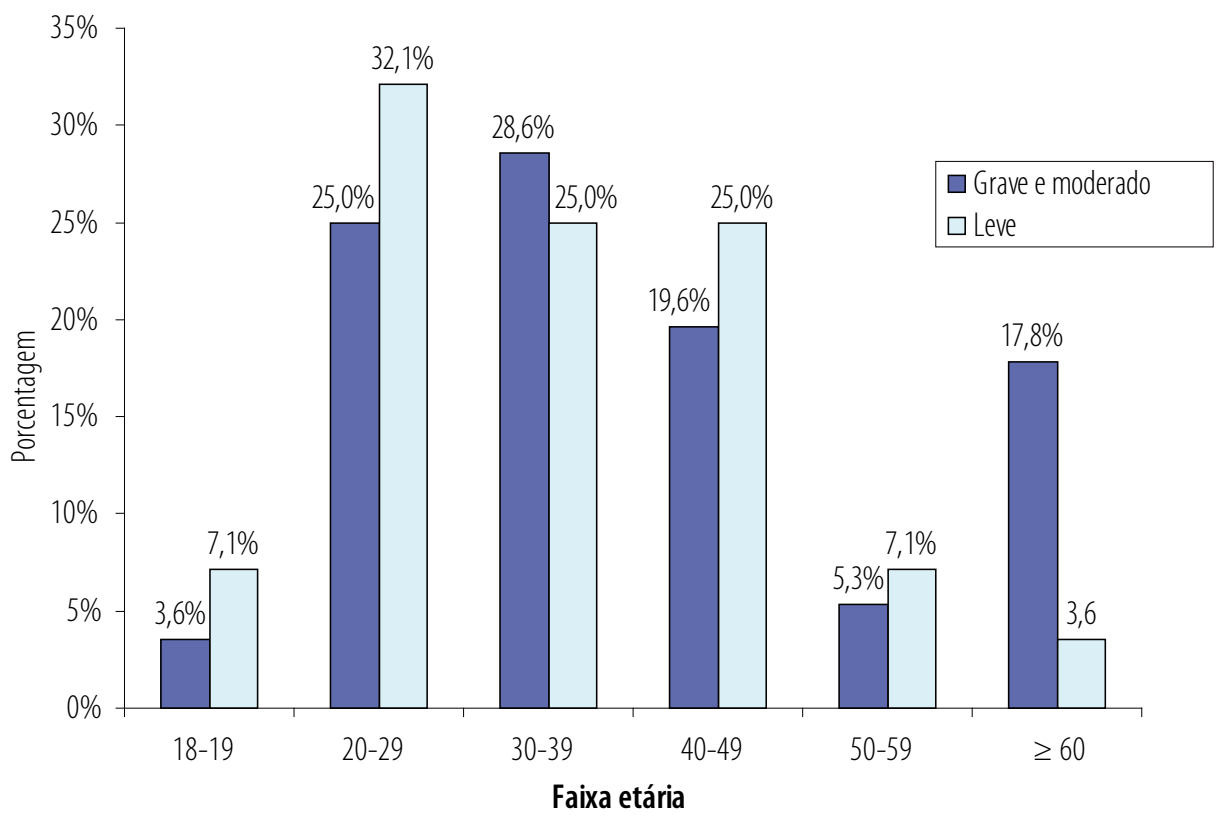

Figura 2 - Distribuição de acordo com a faixa etária dos pacientes com TCE grave, moderado e leve $(n=85)$, selecionados para coleta de urina para dosagem de drogas com idade igual ou superior a 18 anos atendidos na UE do HC da UFU.

Dez por cento dos pacientes envolvidos em acidente de transporte apresentavam exames positivos para cocaína e $4 \%$ para maconha. Dentre os envolvidos em agressão, 29,4\% tinham exames positivos para cocaína e $18,9 \%$ para maconha. A evolução para óbito entre esses pacientes ficou restrita à sua minoria. 


\section{Discussão}

Este estudo revela uma frequência de TCE grave e moderado $(44,8 \%)$ semelhante à de TCE leve no ano de 2002, sendo discordante dos dados da literatura, ${ }^{1} \mathrm{o}$ que se explica pelo fato de o $\mathrm{HC}$ da UFU ser a única referência terciária do SUS em Uberlândia, sendo para lá encaminhados com maior frequência os pacientes graves, e também pelo fato de as unidades de atendimento periférico na cidade absorverem parte da demanda de TCE leve.

Os achados em relação à distribuição semanal da chegada do paciente à UE predominantemente nos fins de semana corroboram os resultados da literatura, ${ }^{6,15,22}$ embora estes dois últimos autores tenham observado um pico de ocorrência na sexta-feira e neste estudo tenha ocorrido no sábado; os fins de semana são momentos para viagens e desafortunadamente também para o consumo de álcool, favorecendo a ocorrência de injúrias. ${ }^{3}$

Quanto à distribuição circadiana, os resultados são condizentes com os da literatura, sendo observado maior número de pacientes com TCE chegando à UE no final da tarde e início da noite, momento do rush e início das atividades de lazer. ${ }^{6,22}$ No período noturno, há maior consumo de bebidas alcoólicas e uso de drogas e maior dificuldade de visualização, fatores determinantes da ocorrência da injúria. ${ }^{3}$

Quanto à idade, foi observado um predomínio de adultos jovens, concordante com a literatura quando se enfoca apenas a população adulta. ${ }^{11,15,20}$ Em nosso estudo, o pico de ocorrência se deu na faixa dos 20 aos 29 anos e dos 30 aos 39 anos, de forma semelhante aos achados de Bowers e Marshall. ${ }^{4}$

Observou-se predomínio do TCE em pessoas do sexo masculino, o que corrobora a maioria dos dados da literatura. ${ }^{6-8,15,23,27}$ Neste estudo, encontrou-se uma relação de 5,95 homens para 1 mulher, enquanto na maioria dos estudos a proporção de homens é de duas a três vezes maior que a de mulheres, ${ }^{6,7,23,27}$ embora haja relato de uma relação de $7,7: 1,0 .{ }^{15,27}$ A proporção de homens aumenta com a severidade do trauma ${ }^{19}$ e este estudo tratou de pacientes com TCE grave e moderado, além do que há maior possibilidade de exposição ocupacional e recreacional dos homens em relação às mulheres; outro fator é o predomínio de homens em acidentes de trânsito e maior consumo de álcool pelas pessoas do sexo masculino.

Observou-se que $64,02 \%$ das causas externas, relacionadas ao TCE grave e moderado, foram por acidentes de transporte, seguidos de queda $(17,27 \%)$ e agressões (16,55\%) (Figura 3), corroborando a maioria dos autores, ${ }^{13,15,16,22,25}$ embora em Medellin et al. ${ }^{27}$ se tenha observado maior número de agressões que quedas, fator relacionado a características das violências locais. Também Colli et al. ${ }^{6}$ no HC da FMRP encontraram uma frequência de quedas e acidentes de trânsito semelhantes, dados também verificados por Jager et al. ${ }^{11}$ Os acidentes de transporte e agressões ocorreram mais frequentemente nas faixas etárias dos 18 aos 59 anos, achado condizente com a literatura ${ }^{16}$, sendo as quedas mais frequentes acima dos 60 anos..$^{6,11,16}$

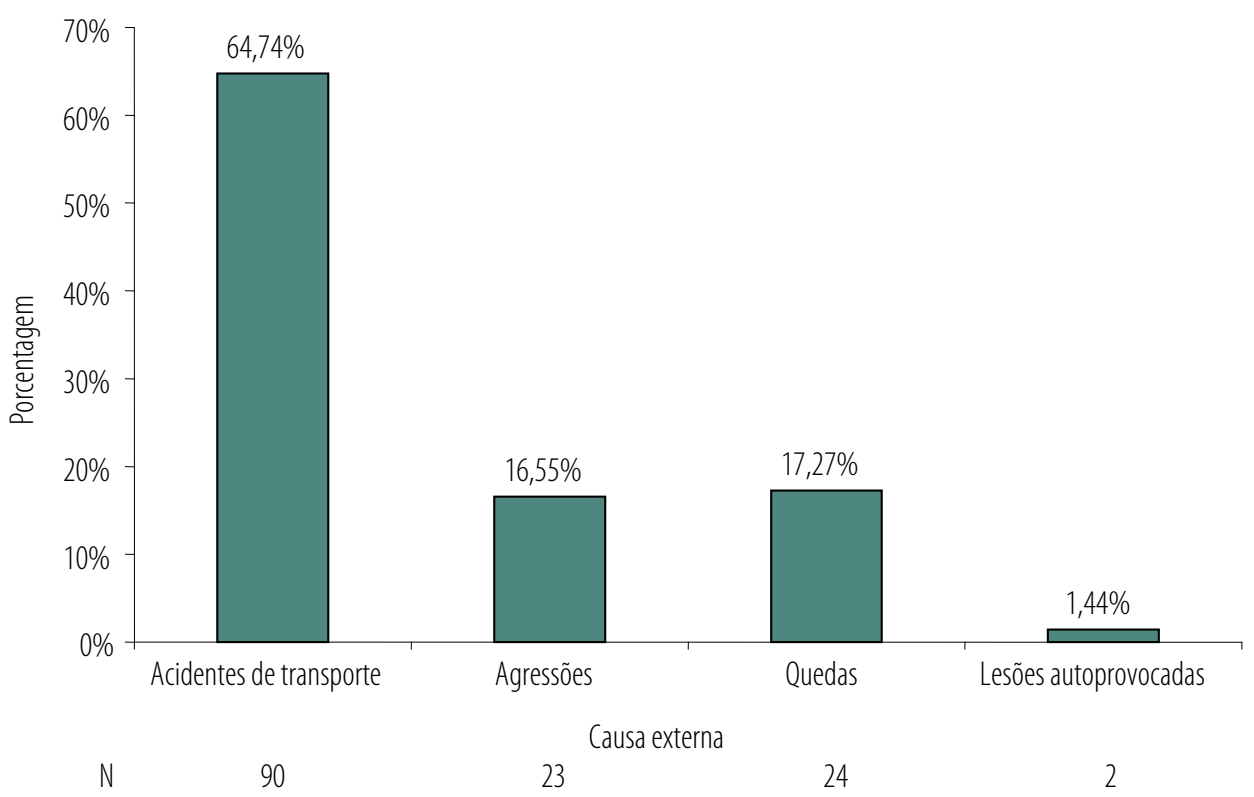

Figura 3 - Distribuição de acordo com as causas externas dos pacientes com TCE grave e moderado $(n=139)$ com idade igual ou superior a 18 anos, atendidos na UE do HC da UFU no ano de 2002. 
Dos acidentes de transporte, $\mathrm{o}$ acidente motociclístico foi o mais frequente $(41,58 \%)$, seguido pelos de pedestres $(34,83 \%)$ e ocupantes de automóvel $(12,36 \%)$. Esses resultados são discordantes dos da literatura em geral, a qual atribui frequência destacada às colisões entre veículos como causa mais frequente, seguida de atropelamento e, em terceiro lugar, de acidentes motociclísticos. ${ }^{6,11,15,16,20}$ Provavelmente, o fato da grande frequência de não uso de capacetes observado neste estudo (30\%) tenha favorecido uma maior ocorrência de acidentes motociclísticos graves nessa população, fato que deve ser melhor avaliado em estudos futuros.

Quanto ao uso de drogas, observou-se que 8,3\% dos pacientes apresentavam exames preliminares positivos para maconha e $13,9 \%$ para cocaína, resultados discordantes da maioria dos autores que encontraram maior frequência de maconha do que de cocaína ${ }^{5,26} \mathrm{e}$ uma frequência de exames positivos para droga superior aos nossos achados. ${ }^{21,28}$ Essas diferenças provavelmente se devem à forma de seleção dos pacientes, ao fato de o objeto de estudo ser apenas o paciente com TCE e às características locais do consumo de drogas. A prevalência de exames positivos no decorrer da semana foi maior nos finais de semana, com pico para cocaína no domingo e de maconha no sábado, com maior prevalência da chegada no período do final da tarde e noite.

Quanto à faixa etária, a maior frequência foi dos 20 aos 29 anos para cocaína e dos 50 aos 59 anos para ma- conha, não sendo observado qualquer exame positivo acima dos 60 anos, dados que corroboram parcialmente outros estudos ${ }^{26,28}$ discordando com maior frequência do uso de maconha em idades mais elevadas, o que não foi significativo, podendo estar associado ao pequeno número de casos encontrado.

Com relação ao sexo, foi observado um maior número de exames positivos no sexo masculino corroborando a literatura. ${ }^{26,28}$ Em relação à causa externa, observou-se predomínio de exames positivos em vítimas de agressão, demonstrando a associação do uso de drogas com os traumas intencionais e com a violência, o que corrobora a literatura. ${ }^{18,26,28}$ (Tabelas 1 e 2).

Este estudo não obteve resultados que evidenciassem qualquer relação entre o uso das drogas e a letalidade no TCE, concordante com Wagner et al. ${ }^{28}$, que encontraram o uso de drogas como sendo fator protetor para a severidade da injúria, sugerindo a necessidade de novos estudos investigando esses achados. A população de pacientes usuários de drogas neste estudo é pequena, não sendo suficiente para qualquer conclusão no que tange à letalidade. Uma vez que não estudamos pacientes que foram a óbito antes de adentrarem ao hospital, este estudo pode também subestimar a verdadeira relação entre óbito e frequência de exames positivos para drogas, sendo concordante quanto à necessidade de mais estudos para elucidar a relação entre uso de cocaína e mortalidade. ${ }^{18}$

\begin{tabular}{|c|c|c|c|c|c|}
\hline \multirow{2}{*}{ Tipos de causa externa } & \multicolumn{2}{|c|}{ Cocaína } & \multirow{2}{*}{$\mathbf{R R}$} & \multirow{2}{*}{ IC 95\% } & \multirow{2}{*}{$\mathbf{p}^{*}$} \\
\hline & Positivo & Negativo & & & \\
\hline Acidente de transporte & 5 & 45 & 1 & - & - \\
\hline Agressão & 5 & 17 & 2,227 & $0,73-7,06$ & 0,26 \\
\hline Quedas & 0 & 21 & 0 & - & 0,57 \\
\hline Outras & 1 & 0 & 10,0 & $4,35-22,97$ & 0,11 \\
\hline
\end{tabular}

* Teste exato de Fisher.

Tabela 2 - Distribuição dos pacientes de acordo com a causa externa $(n=84)$ em relação ao resultado positivo ou negativo dos testes para deteç̧ão de maconha na urina

\begin{tabular}{|c|c|c|c|c|c|}
\hline \multirow{2}{*}{ Tipos de causa externa } & \multicolumn{2}{|c|}{ Maconha } & \multirow{2}{*}{$\mathbf{R R}$} & \multirow{2}{*}{ IC $95 \%$} & \multirow{2}{*}{$\mathbf{p}^{*}$} \\
\hline & Positivo & Negativo & & & \\
\hline Acidente de transporte & 2 & 48 & 1 & - & - \\
\hline Agressão & 4 & 18 & 4,55 & $0,90-23,0$ & 0,70 \\
\hline Quedas & 1 & 10 & 2,27 & - & 0,46 \\
\hline
\end{tabular}

* Teste exato de Fisher. 


\section{Conclusão}

Este estudo, em concordância com outros da literatura, aponta para uma alta prevalência de TCE em indivíduos na faixa etária produtiva. Ressalta-se grande frequência de acidentes de transporte, bem como o grande percentual de óbitos, o que provavelmente representa um alto custo para a sociedade. Assim sendo, ainda que preliminares, estes resultados servem como fundamentação e motivo para projetos de prevenção desse problema de saúde pública, devendo também desencadear medidas para reduzir o impacto negativo do consumo de drogas na saúde.

Este estudo também serve para evidenciar a importância da inclusão da história de abuso de substâncias em análises sobre TCE e encorajar novos estudos, que, se possível, utilizem uma amostra populacional maior e testes mais aprimorados, para que novos subsídios possam ser fornecidos para o controle e prevenção desse trauma e do abuso das substâncias associado a ele.

\section{Referências}

1. Andrade AF, Marino Jr R, Miura FK, Carvalhaes CC, Tarico MA, Lázaro RS, et al. Diagnóstico e conduta no paciente com traumatismo craniencefálico leve. Projeto Diretrizes: Sociedade Brasileira de Neurocirurgia; 2001.

2. Baker SP, O'Neill B, Haddon W Jr, Long WB. The injury severity score: a method for describing patients with multiple injuries and evaluating emergency care. J Trauma. 1974;14(3):187-96.

3. Barss P, Smith GS, Baker SP, Mohan D. Injury prevention: an international perspective epidemiology, surveillance, and policy. New York: Oxford University Press; 1998.

4. Bowers SA, Marshall LF. Outcome in 200 consecutive cases of severe head injury treated in San Diego County: a prospective analysis. Neurosurgery. 1980;6(3):237-42.

5. Brookoff D, Cook CS, Williams C, Mann CS. Testing reckless drivers for cocaine and marijuana. $\mathrm{N}$ Engl $\mathrm{J}$ Med. 1994;331(8):518-22.

6. Colli BO, Sato T, De Oliveira RS, Sassoli VP, Cibantos Filho JS, Manço AR, et al. [Characteristics of the patients with head injury assisted at the Hospital das Clínicas of the Ribeirão Preto Medical School]. Arq Neuropsiquiatr. 1997;55(1):91-100.

7. Frankowski RF. Descriptive epidemiologic studies of head injury in the United States: 1974-1984. Adv Psychosom Med. 1986;16:153-72.

8. Gale JL, Dikmen S, Wyler A, Temkin N, McLean A. Head injury in the Pacific Northwest. Neurosurgery. 1983;12(5):487-91.

9. Hawks RL. Consensus report. Drug concentrations and driving impairment. Consensus Development Panel. JAMA. 1985;254(18):2618-21.

10. Hsiang JN, Yeung T, Yu AL, Poon WS. High-risk mild head injury. J Neurosurg.1997;87(2):234-8.

11. Jager TE, Weiss HB, Coben JH, Pepe PE. Traumatic brain injuries evaluated in U.S. emergency departments, 19921994. Acad Emerg Med. 2000;7(2):134-40.
12. Jagger J, Fife D, Vernberg K, Jane JA. Effect of alcohol intoxication on the diagnosis and apparent severity of brain injury. Neurosurgery. 1984;15(3):303-6.

13. Jennett $B$, MacMillan $\mathrm{R}$. Epidemiology of head injury. $\mathrm{Br}$ Med J (Clin Res Ed). 1981;282(6258):101-4.

14. Teasdale G, Jennett B. Assessment of coma and impaired consciousness. A practical scale. Lancet. 1974;2(7872):81-4.

15. Klauber MR, Marshall LF, Barrett-Connor E, Bowers SA. Prospective study of patients hospitalized with head injury in San Diego County, 1978. Neurosurgery. 1981;9(3):236-41.

16. Kraus JF, Black MA, Hessol N, Ley P, Rokaw W, Sullivan $\mathrm{C}$, et al. The incidence of acute brain injury and serious impairment in a defined population. Am J Epidemiol. 1984;119(2):186-201.

17. Kraus JF, McArthur DL. Epidemiologic aspects of brain injury. Neurol Clin. 1996;14(2):435-50.

18. Lindenbaum GA, Carroll SF, Daskal I, Kapusnick R. Patterns of alcohol and drug abuse in an urban trauma center: the increasing role of cocaine abuse. J Trauma. 1989;29(12):1654-8.

19. Masson F. [Epidemiology of severe cranial injuries]. Ann $\mathrm{Fr}$ Anesth Reanim. 2000;19(4):261-9.

20. Mazartins ET, Bruggemann M, Coutinho M. Estudo prospectivo de 200 casos de TCE grave na grande Florianópolis. Rev Bras Terap Intens. 1997;9(4):175-80.

21. Marzuk PM, Tardiff K, Leon AC, Stajic M, Morgan EB, Mann JJ. Prevalence of recent cocaine use among motor vehicle fatalities in New York City. JAMA. 1990;263(2):250-6.

22. Masini M. Perfil epidemiológico do traumatismo cranioencefálico no Distrito Federal [tese]. São Paulo: Escola Paulista de Medicina, Universidade Federal de São Paulo; 1994.

23. Obembe A, Fagbayi A. Road traffic accidents in Kaduna metropolis: a-3 month survey. East Afr Med J. 1988;65(9):572-7.

24. Peixoto HCG, Souza ML. Anos potenciais de vida perdidos e os padrões de mortalidade por sexo em Santa Catarina, 1995. Informe Epidemiológico do SUS. 1999;8(2):47-52.

25. Rimel RW, Giordani B, Barth JT, Jane JA. Moderate head injury: completing the clinical spectrum of brain trauma. Neurosurgery. 1982;11(3):344-51.

26. Rivara FP, Mueller BA, Fligner CL, Luna G, Raisys VA, Copass $\mathrm{M}$, et al. Drug use in trauma victims. J Trauma. 1989;29(4):462-70.

27. Turizo RA, Giraldo CA. Mortalidad por trauma encefalocraneano. Instituto de Medicina Legal, Dseccional Medellín. Medellín: Ed Colombia; 1985. p. 291-300.

28. Wagner AK, Sasser HC, Hammond FM, Wiercisiewski $\mathrm{D}$, Alexander J. Intentional traumatic brain injury: epidemiology, risk factors, and associations with injury severity and mortality. J Trauma. 2000;49(3):404-10.

29. Weiss RD, Gawin FH. Protracted elimination of cocaine metabolites in long-term, high-dose cocaine abusers. Am J Med. 1988;85(6):879-80.

30. Youmans JR. Neurological surgery: a comprehensive reference guide to the diagnosis and management of neurosurgical problems. Philadelphia: Saunders; 1996. p. 1531-2491.

\footnotetext{
Endereço para correspondência

José Weber Vieira de Faria

Rua Tiradentes, 250, ap. 805, Fundinho

38400-250 - Uberlândia, MG

Telefone: $(+55$ 34) 9979-7309

E-mail: weberneuro@yahoo.com.br
} 